\title{
Gesture, meaning-making, and embodiment: Second language learning in an elementary classroom
}

\section{Alessandro Rosborough}

Abstract: The purpose of the present study was to investigate the mediational role of gesture and body movement/positioning between a teacher and an English language learner in a second-grade classroom. Responding to Thibault's (2011) call for understanding language through whole-body sense making, aspects of gesture and body positioning were analyzed for their role as mediational tools for meaning making during a math assignment. Analysis of the teacher-student dyad provides insight as to how they moved from simply exchanging answers to using positions and gestures to embody meaning and feelings, thus establishing strategic ways to solve communication problems in the future. A shift to embodying the communication task provided new meanings not previously afforded while sitting at a desk. Combining a Gibsonian (1979) ecological perspective with Vygotskian $(1978,1986)$ sociocultural theory provides a way to view the role of embodiment in the social practice of second language learning (van Lier, 2004). Findings provide evidence that gesture along with bodily positions and [inter]actions play a central role in this dyadic meaning-making experience. The data demonstrate the interactive nature of the semiotic resources of the activity (i.e., speech, gesture/hands, math graph, whiteboard), with their materialized bodily/speech-voiced acts coinciding with Thibault's (2004, 2011) explanation of human meaning-making activity as a hybrid phenomenon that includes a cross-coupled relationship between semiotic affordances and physical-material body activity. This perspective embraces Vygotsky's (1978, 1997a) view of dialectical development including the importance of psychological and materialized-physical tools such as gesture in dealing with language learning processes (McNeill, 2012).

Key words: gesture, embodiment, mimesis, second language learning 


\section{Second language learning in an elementary classroom}

What constitutes appropriate pedagogy and practices for second language learners is a ubiquitous question in the field of education, continually addressed in the past as well as the present. Some of the issues questioned concern how to handle multiple languages in one classroom. In dealing with minority language issues, many United States school districts have turned to protocols and models for dealing with diverse languages. These curricula are primarily based on traditional language perspectives that include (1) describing language as having correct standardized modalities, (2) positioning computational input and output exchanges as the crucial means to learning, and (3) treating language as an autonomous system that is independent from other aspects of life. From a non-critical perspective, such outlooks and models are convenient in that they place language as a systematic code of symbols quite separable from meaning making.

This provides early elementary grade curriculum writers and teachers convenient ways to instruct and model language-learning objectives as systematic tasks, based largely on the premise that following systemic language patterns will lead to successful application on summative tests. As a result, when teachers consider second language learners (SLLs) in their classroom, lesson plans are often created and implemented using the perspective that adding a few accommodations or modifications will fulfill the requirement for meeting second language learning needs. However, this view of working with SLLs reduces language to just a "medium" or "text" that is separable from its meaning or material expression (Thibault, 2011). In this mindset, "text," oral or written, is only instantiated to the acknowledged measurement level of the particular protocol or pedagogical program selected for a given site. As a result, a model calling for exact answers strongly overshadows important processes of language learning, including the meaning-making process among participants. Consequently, language is often separated from its embodied and material dynamics and replaced with a "language as a code" perspective in many second language classroom settings (van Lier, 2004).

The instantiation and legitimacy of prescripted educational curricula often fail to acknowledge that humans, especially children, position their decision-making and meaning-making abilities through perspectives and perceptions that are affected and transformed through corporeal positionings of their embodied mind. In particular, gesture has been afforded merely a cursory space on educational scripts, leaving teachers and their classroom 
communities very little understanding for how gesticulations manifest action and imagery as part of the process of speaking, communicating, and meaning making in a second language.

Considerable attention to gesture is needed if we are to understand the meaning-making processes that occur in second language learning (Gullberg, 1998; Gullberg \& McCafferty, 2008; McCafferty \& Stam, 2008). Considering children as fully vested agents in their learning and meaning-making paths, and understanding the place of embodied experiences in their new language settings provide additional insights for language in use, which include the second language learners' authentic discourse and actuations that occur in the classroom.

Actuations have to do with learning through perception of signs, artifacts, or texts available in an ecology, acting with them, and developing mediational [inter]actions with these "tools" to create new real-world meanings in the situated moment. Merleau-Ponty (1962) and McNeill (2012) described this as "inhabiting" a language-meaning making by living in and through a language, including the use of imagery, speech, and thoughts. To understand how a second language learner inhabits and interacts in, with, and through a language, a more thorough understanding of learning through the body, including corporeal interactions, must be considered. The role of embodiment, including gesture, as a mediational affordance in understanding language learning interactions needs a more comprehensive exploration if we are to understand the meaning-making processes between teachers and young ELLs.

\section{Review of literature}

\section{Gesture, and Sociocultural Learning}

Within the last decade, gesture has been included as a part of the list of recommended teaching tools for English language learners. Educational programs and protocols suggest the use of gesture as a part of the lesson planning process and recommend that teachers include it to facilitate comprehension (Echevarria \& Graves, 2011; Echevarria, Vogt, \& Short, 2000). A superficial emphasis on gesture use reduces it to just another mode to create language defined by its conduit or computational functions as an input-output exchange system. Such a definition perpetuates the notion that language is simply a conduit for exchanging pieces of information. It also perpetuates second language curricula as little more than simple protocols, with recommended lists of practices for working with second language learners in the classroom. 
Although use of gesture has been added to best practice lists, there is little to no discussion on how to use this "strategy." Little effort is made to further examine how gesture is implemented effectively with little in-depth discussion concerning why teachers should attend to corporeal learning. Typically, explanations are based on the false notion that gestures reinforce and clarify meanings already presented in speech alone (McNeill, 2012). Such stances neglect to consider the contributions of imagery and analogous physical movements to cognition, language learning, and information acquisition while working with people and socially constructed artifacts.

The phenomena occurring between teachers and students during second language acquisition may be more readily explicated using Vygotsky's (1997a) sociocultural theory (Lantolf, 2000; Wertsch, 1991). Indeed, Vygotsky (1986) viewed gesture as forward-oriented languaging, a material carrier of meaning that complements and also precedes verbal meaning making between a child and caretaker. Although not referring specifically to second language learning, Vygotsky commented that gesture was an indivisible part of learning and thinking through language. Research using Vygotskian sociocultural theory for understanding second language issues has been successfully applied (Kramsch, 2008; Lantolf, 2000; Lantolf \& Johnson, 2007; Lantolf \& Poehner, 2008; Lantolf \& Thorne, 2006; Swain \& Deters, 2007).

Along with his explication of gesture, Vygotsky (1986) extended the concept that understanding children's speech meaning is found only through understanding of the word's function during mediated specific activities. Vygotsky (1978) stressed that during the activity, in a microgenetic and materialized form, caretakers (in this case teachers) can see the how and why of "changes" (i.e., learning and development) in a student's experience. According to Vygotsky (1997b), the key to understanding meaning making of humans is through mediational interactions with psychological and physical tools. This occurs in materialized states, including activities with affordances or any semiotic signs found in their ecology (Gibson, 1979).

Gibson's (1979) ecological learning perspective considered meaning making as an active and interdependent relationship including the learner, his or her perception abilities, and the affordances (e.g., objects, artifacts, tools) in an environment. His explication of visual observation and world understanding included his hypothesis termed ground theory (p. 148). In this hypothesis, the interrelationships of places, objects, and perception are all part of the "layout of surfaces" in connection with the ground (i.e., a physical 
terrestrial surface with horizons, vanishing points, and so forth). Comprehension of the interrelationship of these surfaces is ultimately understood only through self-perception, since an observer's perspective changes according to his or her physical movement and distance from the surfaces.

Concerning second language learning, van Lier $(1996,2004,2008)$ applied Gibson's interrelational ecological approach and Vygotskian concepts of mediational learning and internalization, terming it ecosocial language learning. Viewing second language learning as a socially and physically situated activity places gesture as an important affordance of an environment and an important resource for teachers and students to recognize in their learning (Kida, 2008; Kramsch, 2008; Lazaraton, 2004; Sime, 2006). The emphasis concerning second language learning in relation to ecologically situated affordances coincides with the claim of William James (1890) and Donald (2001) that conscious ideas and images are always owned, and this owning is highly physical and body-based. Donald expressly claimed that body-based consciousness and learning is the "farthest thing in the world from a rational or linguistic sense of owning" (p. 134). Coupling this type of learning with communication, Donald viewed languaging through the lens of mimesis: a type of imitative acting between participants manifested through pantomime, gestures, shared attention, and other ritualized shared behaviors.

\section{Mimesis and Second Language Learning}

Extending the work of Donald (2001), McCafferty (2008a) defined mimesis specific to second language learning as having to do with imitation, representation, and image as a learner pantomimes, imitates, gestures, and shares attention with another participant. In this case, mimesis is not a copy of a representation but more of a "construal" or "interpretation" of it (Goodman, 1968, p. 9). McCafferty presented an argument for mimesis as a foundational grounding for second language teaching and learning stating, "Any truly comprehensive theory of SLA will need to move beyond the mind-body dualism that currently pervades the field and take into account our material experience in the world as an aspect of living and learning" (p. 164). Similar to Donald but specific to second language learning, McCafferty stressed that owning conscious ideas and learning has to do with the physical manifestations of ideas and images as a form of understanding and meaning making by the L2 learner (see also Haught \& McCafferty, 2008). 
Differing from a structural language and codified perspective, emblematic and spontaneous gestures are important to study in education and second language learning because they typically originate from a personal experience in the world and thus are situationally meaningful and analogously attached to the ecosocial environment (Tomasello, 2008). Evidence of gesture use in a forward-oriented appropriation and ecosocial process was found in McCafferty's (1998, 2008b) studies of Japanese speakers learning English in the United States and Faraco and Kida's (2008) analysis of didactic and dyadic situations in L2 language classrooms. In McCafferty's studies, Japanese English learners showed they were appropriating the surrounding American culture by displaying gestures not traditionally used in Japan. Faraco and Kida evaluated both positive and problematic uses of gestures in multiple settings. They found that gestures facilitated reactions and additionally reactions to the reactions between participants, dependent on the extent of the linguistic focus. When the lesson centered succinctly on linguistic form, the teacher did not initially use gestures. However, when the classroom conversation moved towards meaning making, gesture was an important part of the discourse among the participants. In both studies, participants displayed gestures as an essential affordance for their meaning-making processes with their new second language.

\section{Vygotsky's Non-Traditional Psychology and Corporeality}

Vygotsky (1978) promoted activity in a concept he termed the zone of proximal development (ZPD), an assessment of levels at which one can engage, learn, and develop with appropriate support. In educational terms the ZPD can be viewed as an ecosocial approach, since it is a perceptible and tangible situation in which conditions can be overtly observed and tools or signs can be physically and psychologically constructed, shared, and materially "grounded" among participants.

Complementing Vygotsky's ZPD is the related notion promoted by Del Rio and Alvarez (2007) and Zazzo (1968) of functional anticipation, an orientation towards forward use and development rather than just the recall of what is being taught. Building off of Vygotskian theory, Valsiner (1997) addressed dyadic co-construction and future orientation as internalization: "a constant forward-oriented construction of signs that bring over from the extrapersonal (social) world of the person to the intrapersonal subjective world semiotically encoded experiences" (p. 246). This statement is not limited to a shared historic background or a particular culture, but includes the creation of intersubjective experiences as generated by participants using the affordances 
(i.e., artifacts and tools) situated in their ecology. Vygotsky (1998) claimed that intersubjective relationships can be understood through materialization using the embodied-situated mind in learning directions displaying projections towards future development.

Wertsch (1998) explained Vygotsky's (1978) future orientation, including the concept of internalization as a process of appropriation. Included in this concept is the understanding that acquiring "static" answers does not provide sufficient insights for projections of learning and for further projections of how a learner can use and change tools in differing circumstances. Without incorporation of embodied ecological experience, students and teachers are often left with simple transmissions of pieces of language. Such learning paths limit ELLs' understanding of how to learn, act, and inhabit a language.

\section{Methodology}

Using a Vygotskian sociocultural theoretical framework, this study probed the use of embodiment, including gesture, by a teacher and student in an elementary classroom with sheltered English language learning. The purpose of the study was to explore the use of gestures as mediational tools for teaching and learning a second language. Differing from mainstream educational views of the role and function of gesture in the classroom (Rosborough, 2012), this study considers gesture as a fully integrated aspect of language and communication to be analyzed in order to understand and interpret the meaning-making processes between a teacher and students in an L2 setting.

Implementing a qualitative methodology to understand a classroom's collective and individual meaning-making experience involving language, communication, and social contexts, the researcher collected data for 28 complete school days in a second grade classroom during the spring months. The excerpts selected for this paper are part of this larger study which utilized a variety of data sources: video recording by multiple cameras, observation and field notes, classroom documents including multiple assessments, and two interviews with the teacher.

\section{Setting}

This study was performed in a sheltered English second grade classroom located in an urban southwest U.S. city. Located near the center of the 
city, the school qualifies as a Title I school, with a high majority of students qualifying for reduced price lunch. The classroom site was purposefully selected because it was the only sheltered-English classroom in an English full-immersion school. The classroom was "sheltered" in that all students were ELLs, and the lessons were prepared with modifications and adaptations specific to this population's needs. Placement decisions for students were based on language abilities, ranging from emerging to intermediate levels. This classroom paralleled "mainstream" curriculum, using similar educational programs and assessments.

\section{Participants}

The teacher, referred to with the pseudonym Mrs. Dee, was a female English speaker with self-taught beginning Spanish-speaking abilities gained during 19 years as an elementary educator. For 17 of the 19 years she had been an ESL instructor of second grade. Her formal education included an MA in education and TESOL certification; her thesis had emphasized literacy and interaction in learning.

The student participants were 19 bilingual students. The languages they spoke were Spanish, Bengali, Arabic, and Tagalog, with Spanish being predominant. In the morning the class consisted of 18 students, joined for the afternoon by a student whose dominant language was Tagalog. The characteristics of this program met the needs of the study with its focus on teaching and learning in English as a second language for the students. A number of the students were recent immigrants to the U.S., and all of them had been identified and labeled as limited English proficient (LEP) by government standards.

The student selected to be studied for this paper was a female Spanish speaker, referred to with the pseudonym Liliana, who had participated in the class almost the entire year. She had missed preliminary standardized tests in September, but was tested in December for reading connected texts and individual words, as well as for spelling. Her scores placed her in the lowest $1 / 6$ th of the class. Previous school records for her did not exist, and school officials believed she had had no previous years of schooling in the United States.

\section{Data Collection}

Classroom interactions between the teacher and students were recorded using three video cameras. Two cameras were placed in the corners of the room to provide wide-angle views of the face-to-face classroom interactions. 
One mobile camera with zoom-specific abilities was placed on a monopod to record close-up interactions and serve as back-up reception. The teacher wore a wireless microphone with reception to the stationary cameras. According to opportunity and activity, a second wireless microphone was placed near some student-to-student dyads. Altogether 28 full days were recorded for a total of 131 hours. For this paper, one excerpt was selected from 80 hours of analyzed video.

Observation of the students was the central role of the researcher. Although some conversations and friendly relationship building occurred as a natural result of an adult being in the room with children seven to eight years of age, interactions were minimal, and no tests, strategies, techniques, artifacts, or experiments were performed or instituted by the researcher. The teacher and students were unaware of the foci of the study. The data include a variety of authentic tasks that occur in most elementary classrooms, and the teacher's lessons and interactions with the students were viewed as being natural with no test intrusion.

\section{Data Analysis: Microgenetic Method}

Vygotsky (1978) argued that a genetic view of learning and development is the only way to understand the inner workings involved with acquiring higher mental functions. Genesis, in Vygotskian terms, has to do with the mediational roles of culture and history in constructing higher forms of thinking. Higher functions are historic, meaning that they are constructed or developed through participation with the social world (Daniels, Cole, \& Wertsch, 2007; Lantolf \& Thorne, 2006; Vygotsky, 1978, 1981; Wertsch, 1985, 1991).

A microgenetic approach to analysis focuses on processes of development over short periods of time, allowing the researcher to examine social and individual activities from the genetic roots onward. Microgenesis is associated with observing and explaining an event or activity that is being transformed by some mediational tool or means, including people, which in turn influences the transformation of the individual's potential to further the activity ontogenetically (Wells, 1999). This study used a genetic approach, with observation, video recording, note-taking, transcriptions, interviews, and review of the video allowing the researcher to follow the construction or emergence of teacher and student gesture forms as the two made meaning in the second language (L2).

The analysis method was based on identifying language challenges in which problem-centered learning was evident. These challenges were se- 
lected as examples of the student being pushed beyond her self-regulated abilities. The excerpt selected for analysis displays the repeating themes of (1) joint attention and shared intentionality for materialized language, (2) intersubjectivity, and (3) a forward-oriented transformation or internalization/ appropriation process. These themes were all situated in the experience of the student learning a challenging concept. Specific to a microgenetic analysis of gesture, a modified McNeillian (1992) data coding procedure was used (see Transcript Coding in Appendix A).

As commonly understood, gestures are polysemous, such as being deictic/indexical with a beat at the same time. Performing an exact count of the multiple gestures in these and other excerpts would be illusive and unproductive. As an alternative to gesture counts, a microgenetic analysis of gestures provides what Geertz (1973) describes as "thick description" of information and processes between participants. This type of descriptive analysis cannot be established by trying to number and categorize each spontaneous hand movement in relation to the speech and communicative patterns between participants. Instead, gestures are coded for both their materialized form and their meaning in holistic acting functions.

\section{Transcript Coding}

Coding of the transcriptions included both the speech and gestures of participants. Coding and transcription style was based on a variation of McNeill's (1992) verbal/gesture method. The speech transcription is in regular type, and the gesture description is in italics. Coding details are provided in Appendix A. The five gesture types classified by McNeill were used in analyzing the classroom activities: beats, iconics, deictics, metaphors, and emblems. The gesture or stroke phase, placed in brackets with the stroke in bold, was also viewed for any additional clarity or meaning it provided to the situation. Strokes in gesture often anticipate and synchronize in a unified process with the most salient points of speech, centered between a preparation movement and a retraction movement. The entire gesture movement may be significant, or at times only the stroke may be carrying a message (Kendon, 1994; McNeill, 1992). Inclusion of the entire stroke phase was decided on an individual gesticulation basis depending on the context and situation. Bodily movement and positioning in relation to the classroom also complements the verbal transcription.

ELAN and Quicktime software were used to view and create transcripts. Both items of software allowed for a video window to be displayed along with 
a text format to transcribe speech and actions. They also provided a means to adjust the speed of the video, which allowed the researcher to capture both speech and gesture movements that were not as readily identifiable in real time. Micro-analysis of gestures in the video was conducted at $1 / 10$ th of a second.

\section{Findings}

\section{A Problem and an Answer}

During Day 15 Mrs. Dee had students working at their desks on a paper and pencil activity answering a variety of questions written on the whiteboard. As she roamed among the desks assisting students, she saw Liliana struggling to answer one of the math problems using a data chart titled "Hands and Fingers." A one-on-one interaction between Mrs. Dee and Liliana ensued, and both participants turned to an embodied gesture process to address the challenging problem. At the beginning of the interaction, Mrs. Dee asked Liliana to identify the title of the problem, but Liliana was unable to recognize and state it.

\section{Excerpt 1 Part 1}

1M.1 Day 15 (0:15:40) (0:16:20)

03 Mrs. Dee: It's actually "Hands," "Hands and Fingers" // [So look at your hand]

Mrs. Dee: Points to space over paper

04 Liliana: Puts LH palm up, fingers extended, over the paper on the desk with the RH holding the pencil

05 Mrs. Dee: Okay, [how many hands is this $(/ / / /)$ ?]

Grabs Liliana's LH wrist and shakes the hand in front of student at face level with three beats

06 Liliana: One

Liliana: Looks at palm of hand with fingers extended

07 Mrs. Dee: [One hand] How many fingers?

Squeezes Liliana's hand twice around wrist and releases her hand

08 Liliana: [Five]

Hand is still held up, fingers no longer extended but forming a closing/ grasping shape

09 Mrs. Dee: Now look up at the chart. Do you see one hand, five fingers? ////// Do you see that Liliana?

10 Liliana: [//// Because, I know what's one plus // I know the answer. Number 3] 
LH left up with five fingers extended and palm facing self, from when Mrs. Dee holds it up - no stroke or movement

11 Mrs. Dee: Why do you say that?

12 Liliana: Shrugs shoulders up and down, with LH still up in the air with elbow resting on desk

13 Mrs. Dee: You're right, but you have to be able to explain to me why you said that.

14 Liliana: [Because the number]

Looks at hand, LH palm rotated out over desk and then opens palm towards her face

15 [The reason is that there's $\mathbf{1}$ hand], [1, and there's $\mathbf{5}$ fingers], it can get to 15 .

Puts LH back up closer to face level with palm towards her; shakes and points (deictic gesture) to the LH palm with $\mathrm{RH}$ index finger while holding a pencil. Switches deictic gesture pointing at her $L H$ palm from $R H$ finger to end of pencil. Runs the length of the pencil up to the tips of the extended LH fingers. Quickly grasps the pencil when she finishes her statement.

16 Mrs. Dee: [How does it get to 15]?

$B H$ open palm facing up question/presentation gesture

17 Liliana: mmmm, I don't know.

18 Mrs. Dee: Well, let's come up and look at it because, you're right but you've got to be able to explain it to me. (17:00)

Mrs. Dee and Liliana get up and move from desk to chart on the whiteboard.

At the beginning of the dialogue, Mrs. Dee urged Liliana to share her palm with deictic pointing and holding of the wrist, which resulted in joint attention towards Liliana's palms (Lines 6, 7, 8, 14, \& 15). Liliana's hands, the object of their attention, provided a materialized indication of their interpersonal attention and communication. Of particular interest is Line 16, where Liliana was asked how she came up with the answer. When she shrugged her shoulders and said she didn't know, Mrs. Dee switched her emphasis on receiving a spoken answer to an embodied modality to support how the answer was created. Such a question required an unpacking of steps, exemplifying an answer requiring more language and perhaps a greater cognitive challenge.

\section{A Meaning-Making Process}

Mrs. Dee and Liliana moved closer to the board. Of note is a shift from some hand use to actual proximity and hand-as-artifact use near the board. As new spaces were provided, both participants had more room to physi- 
cally instantiate the potential meaning-making process in explaining the construction of the answer.

Excerpt 1 Part 2 - Near the board

1M.1 Day 15 (15:40)

19 Mrs. Dee: One hand, show me one hand. [How many fingers are on that hand?]

Mrs. Dee: RH deictic point to the chart and haptic touch to each part. Touches the number 1, the words "hand" and "fingers," and the number 5 on the chart.

Liliana: Brings left hand, palm up with fingers extended, out in front of her just below shoulder level

20 Liliana: [5]

LH palm up with all fingers extended out and away from each other

21 Mrs. Dee: Okay/ let's look/ [So 1 hand] [5 fingers.] [The next number under hands is what?] On the chart has what number?

Index/deictic pointing to word "hand" on chart, then moves to next column and points/touches number 5. She moves her finger in a downward motion from the word at the top to the number she states.

22 Mrs. Dee: [The next number under hands is what?]

Deictic point and haptic touch by $R H$ index finger back to the hands column one level below the 1- moves RH index finger to fingers column with deictic and haptic gesture

23 Liliana: 2

24 Mrs. Dee: So show me [2 hands].

25 Liliana: Lifts $B H$ up, palm facing towards the board in the same direction she is looking. Mrs. Dee is to her right

26 [And how many fingers does the chart say there are?]

Mrs. Dee: RH index finger continues to point to the number " 2 " and then moves hand to the next column to the number "10"

Liliana: Continues to hold up two hands with palms facing the chart at chest level extended away from body at chest level

27 Liliana: [/ 10]

BH palms momentarily brought down a few inches with palms facing ground and then brought back up facing the board upon verbally answering the question

28 Mrs. Dee: Is that right? Okay.

29 Liliana: [I have 2 hands, I think there's 10 fingers.]

Turns $B H$ palm up while answering the question and then faces them back towards the chart 
30 Mrs. Dee: Okay, so that's right. [So if you have $\mathbf{2}$ hands, you have 10 fingers]/ according to this chart $\backslash /$.

$R H$ index finger performing a deictic and haptic pointing and touching of the two numbers on the chart

[What's the next number?]

RH index finger points back to the hands column by the 2 and then moves to and touches the number 3

31 Liliana: 3

RH fingers still extending but now perpendicular to the chart and her body, with RH fingers extended towards the chart; BHs motionless-no stroke

32 Mrs. Dee: [3 what]?

$R H$ index finger goes to the top of the column and points and touches "hands"

33 Liliana: Hands

34 Mrs. Dee: [Want to borrow my hand?]

Places LH palm up, adjacent to Liliana's two hands with BHs palm down, right hand index finger continuing to point to the 3.

35 Liliana: Shakes head up and down "yes"

36 Mrs. Dee: Okay. [So 3 hands], [how many fingers?]

Points and touches with RH index finger "hands" in the left column, then moves to the right column and points/touches blank space under the "fingers" column

37 Mrs. Dee: So 3 hands, [how many fingers?]

Points with $R H$ index finger to the blank part of the table

38 Liliana: [0]

Brings in all extended 10 fingers and closes BHs (answer space was blank)

The teacher and student continue the dialogue with Liliana identifying 15 fingers for the Fingers column and the answer as "3." In Line 53, Mrs. Dee ends the dialogue sequence with an effort to reinforce what was learned in the task.

Skip to 53

53 Mrs. D: So [when you see charts like this]

$R H$ index finger points to "hands" in left column; all fingers extend with open palm towards the chart, hand waved in a circular motion over the entire chart three times.

[sometimes you can use]

Metaphoric gesture. BHs in front of and between the student and teacher; palms up, fingers extended; makes grasping movements from open palm 
to clenching fists with hands in front of self and student with palm up, fingers extended

[your own self to figure it out].

Using a presentation gesture returns BHs from grasping and fisted to two open palm gestures with fingers. Changing gesture to iconic circles BHs, up and down from shoulder to waist level in a circular motion around the torso of her body. Entire gesture unit finishes with another invitation/ presentation BH palms up gesture with beats, towards S3.

All right. So go circle that number. (19:20)

The use of gestures, those that were spontaneous and some that may have been part of a repertoire from past teaching experiences, provided an imagistic modality not only for coming up with the answer but for extending it towards overtly teaching toward a learning strategy (Line 53) that could become a conscious part of how the student solves problems in the future. In this effort, bodily positionings and gestures assisted in transforming the task to extend beyond only getting the answer right.

To meet the demands of the task, additional support was needed in helping Liliana to understand the "how to solve" strategy. Introducing the palm for Liliana provided a mimetic tool that transformed the activity by offering her an example of how she could self-regulate the solution steps to the math problem in the future. However, the dialogue at the desk and the use of the palms did not seem sufficient in supporting Liliana to produce an explanation for her correct answer.

It is of interest to note that Liliana's change in palm direction embodied her own voice as she imitated Mrs. Dee's suggestions. The new palm direction provided a germinating potential for internalization of embodied learning. Liliana was introducing her own volition and orientation as she initially mimicked Mrs. Dee's hand use but changed her imitation to demonstrate a new orientation of the hands-as-artifacts strategy by using them differently with a new intentionality of solving the problem rather than just coming up with an answer. The materialized strategy heightened accessibility as the participants' interaction extended beyond a simple conduit of speech exchange of information.

In review and interview concerning this video clip, Mrs. Dee identified and labeled it as a "teachable moment." Her familiarity with this student as well as her multiple years of experience provided Mrs. Dee with the knowledge that oral instruction would not always be as effective as participating in an 
embodied practice. Mrs. Dee expressed the importance of deviating from a lesson at the right moment to meet the needs of her students, stating that teachable moments occur, and she wanted to take advantage of them (Interview 1). When Liliana struggled to identify the title of the problem, this experienced teacher recognized the common SLL dilemma that "simplified vocabulary" or a preplanned intervention would be inefficient to develop the concept. She realized that an advanced dialogue would be required to explain the concept of how the answer was constructed and how using bodily strategies for problem solving would be helpful in the future.

In this situation, ecologically situated affordances took precedence in mediating the objective of learning. Instead of relying on typical paper-pencil and simplified oral explanations or basically conventional protocols with comprehensible input, the teacher took a more open path of learning, allowing for the use of affordances with novel possibilities for learning.

In this case, proximity to the board provided a space for interaction for Liliana using her hands and fingers; physical interaction using the board was an affordance beyond mere visual perception of it from the desk. Given the space, Liliana was able to extend her hands and fingers, as deictic and haptic gestures, to create visible and materialized regulation of the problem for both participants. With this physical display, Mrs. Dee coordinated her own hand to add potential tools of assistance such as deictics and haptics to solve the problem.

Concerning deictic and iconic gestures, Lines 19-22 provide evidence of Liliana's hands and fingers touching and representing numbers. Using deictic and haptic gestures, Mrs. Dee's pointing led to Liliana's physical coordination with the problem as she presented two hands with the first two columns. Mrs. Dee offered an additional third hand representing the requested answer in the third column (Lines 19-36). As shown in Lines 34-38, Mrs. Dee literally loaned Liliana a hand. Mrs. Dee turned her left hand palm up for Liliana; however, Liliana kept her hands palms down, demonstrating a different pattern from the open palms up position the participants had established earlier in the dialogue (lines 1-18). At this point Liliana's display of hands might no longer have been functioning for joint attention with the teacher but instead were perhaps directed towards her focus of intention - the problem on the board. Upon answering Mrs. Dee's question in Line 29, Liliana momentarily turned her palms back to face herself and her teacher in a shared communicative pattern, but then she returned her two hands to face the board. 
Materialization of the problem continued when Mrs. Dee pointed to the blank that needed to be answered and Liliana responded with "0," demonstrating the answer when her fingers retracted into fists and then dropped down from the chest level (Line 38). This gesture functioned beyond a jointattention experience, providing Liliana a tangible and concrete embodiment to keep track of the multiple figures on the chart. The teacher and student displayed some counting with fingers, but the coordination of Liliana's hands and fingers not only kept track of numbers, but also accounted for the content or labels in the columns "hands" and "fingers."

Findings of this task show how materialization of the numbers and words on the chart involved both interpersonal and intrapersonal processes in obtaining an explanation to the correct answer. Both Mrs. Dee's and Liliana's hands provided a visible manifestation of their cooperative steps in solving the problem. The two participants made meaning through Mrs. Dee's pointing and guiding and Liliana's heightened physical engagement with her hands directed towards the board. Efforts by both participants brought about a coconstructed answer, but at times intentionality on how to use the hands was not the same. The shared intentionality was evident as three hands were used as a mediational tool for solving the problem. However, the different direction of Liliana's palms demonstrates an actual and representational relationship between her palms and the chart, an important key to understanding the student's intentionality focus and meaning-making path.

\section{Discussion}

\section{Use of Gesture as a Mediational Tool}

This study shows how gesture was used mimetically as a mediational tool for a student learning a second language. It afforded the teacher and student both physical and psychological means for constructing a conceptually shared foundation for meaning making together in English. Similar to a recommendation of McCafferty (2008a), mimesis by the teacher provided affordances in the environment such as proximity to the problem, handsas-artifacts, and materialization of the language on the board. Specific to this study, the materialized discourse supported the meaning-making process by affording joint attention, content coordination, and a transformative change in intention during the activity. Mrs. Dee and Liliana experienced a materialized grounding to their new objective as they embodied the problem. It appears that the objective was no longer just a search for the "correct" answer, but a need to understand how to develop the answer and use 
similar strategies in the future. The change from presenting a mere answer to applying more conceptual and overt attention to how to solve the problem created a more cognitively challenging learning experience. Both participants responded by using their bodies and hands in both similar and differing ways to accomplish the new objective.

The investigation of this teacher-student dyad provides evidence for the potential role of embodiment in supporting the meaning-making experience in the classroom. Differing from many typical reductionist approaches to teaching a second language, Mrs. Dee employed a materialized approach where much of the intersubjectivity and interactions occurred mimetically through body positions and hand gestures. The participants' gestures demonstrated joint attention towards an object and comprehension for a shared purpose. The physical embodiment of the task included multiple acts of cohesion between them. In addition, the learning experience was conducted in the student's zone of proximal development, oriented toward future application rather than toward a static answer. Both teacher and student participated in materializing the problem, embodying it physically with hands and fingers, which actually added more representational complexities to their interaction and also provided additional common ground for learning. They made meaning through the actional and representational gestures and embodiment, resulting in a transformation for working within the task.

The physical materialization of the problem allowed for a greater dynamic space for Liliana to transform her thinking and potentially internalize the concept for the future. Such newly created spaces for learning contrast with many simplified intervention responses or systemic scaffolding steps undertaken to help a student obtain the predetermined correct answer. Beyond accomplishing the objective of getting the answer right, the physical movement of the hands and proximity to the problem on the board provided a heightened engagement of the body, transforming the use of gesture from a function of communicative joint attention to a tool of cognition for unpacking the explanation of the solution in English.

Use of Gesture in Actuating and Inhabiting the Language

Considering the differences between engagement at the desk and embodied engagement near the board reveals a more complete unit for acting, thinking, and moving in a second language. As previously mentioned, the new space afforded Liliana a new focus and direction to display her hand gestures. Liliana's novel use of her hands, beyond referential purposes with the teacher, exemplifies Bakhtin's notion of Chronotope, in which differenc- 
es in time and spaces create opportunities for new voices and utterances. The beginnings of efforts to establish a new conscious realization of how to use gestures as a mediational tool for solving future and different problems (Lines 53-54) may be evident, but understanding maturation of how to use these strategies would need further evidence.

The data do suggest that the teacher and student during Excerpt 1 experienced Kida's (2008) concept of "deeper" communication and comprehension, especially the student. The deeper meaning-making experience was facilitated through an embodied hands-as-artifact experience conducive to providing gestural interaction for pedagogical purposes. These multi-modal affordances provided joint attention, content coordination, and heightened participation. Such uses of affordances helped construct a deeper level of intersubjectivity during the meaning-making process. Again, physical embodiment and novel movements were not simply viewed as appropriate steps towards simple comprehension and exchanges of language pieces. Such new actions provided much more than comprehensible input, by including new and diverse ways of looking at the problem - well beyond merely getting the answer right.

\section{Conclusion}

As this paper explains, future orientation or functional anticipation in teaching, learning, and using a second language often contrasts and conflicts with prescribed assistance techniques suggested by multiple curriculum writers and textbooks for teachers working with ELLs. Common to all humans is the need to make meaning, and the materiality of the body plays a central role in social meaning-making activities (Thibault, 2004). Children especially use the manual/gestural modality as a "boot-strapping" function for lexical and meaning-making needs. For young ELLs, embodying cognition with gesture provides mediational support in accessing the demands of the classroom tasks and the demands of content learning. For relatively recent immigrants and young ELLs, embodied learning, including gesture, is an indispensable support in learning a second language.

Meaning making with activity in language includes a conscious focus on the relationship between meaning and realia in a given situation. Language is often defined as a system for communicating meanings (Zlatev, 2007); however, young ELLs in elementary schools do not necessarily have the same conscious access to prior linguistic knowledge in English as their native-Englishspeaking peers or even as older ELLs. They lack the discourse patterns found between teachers and students from a shared cultural background or gained through social identification with more experienced English speakers. Inter- 
actions between speakers of different languages often lack the transparency and shared pragmatic homogenous conventional discourse existing between speakers of the same language. ELLs in an inclusive classroom do not come with the same five, six, or seven years of experience in using English typical of their dominant-English-speaking peers.

Early elementary children's capacity for reflection and the emergence of inner-speech by their eighth year in their first language do provide them with an orientation toward needs and desires. However, certain conceptual knowledge and higher metalinguistic capabilities are not highly developed in their current developmental phase (Vygotsky, 1986). Hence, materialization and embodiment of language can allow an increase in joint attention and shared intentionality with their instructor, particularly in efforts to have the students learn how to internalize or inhabit the new language or concept. Such teaching demands more overt and grounded materialization than simple production of "correct" answers on a worksheet.

Gesture as a ubiquitous affordance in educational settings, for both inter- and intra-personal processes, needs to be thoughtfully considered in understanding how young elementary children learn and develop in their second language classroom. Although many educational programs suggest the inclusion of gesture in teacher best-practice lists, such generalizations for only cursory communicative functions exclude embodied learning, including the role of gesture in thinking and speaking. As humans are always physically situated and embodiment produces and changes cognitive states (Lindblom \& Ziemke, 2007), promoting an understanding of the role of corporeality in learning provides a deeper engagement in the meaning-making processes in educational settings. Many prescripted curriculua and simple modification steps cannot account for the novel needs and agentive abilities humans have for meaning making when facing learning challenges.

\section{References}

Bakhtin, M. (1981). The dialogic imagination (M. Holquist, Ed.; C. Emerson \& M. Holquist, trans.). Austin, TX: University of Texas Press.

Daniels, H., Cole, M., \& Wertsch, J.V. (Eds.). (2007). The Cambridge companion to Vygotsky. New York, NY: Cambridge University Press.

Del Rio, P., \& Alvarez, A. (2007). Inside and outside the zone of proximal development: An ecofunctional reading of Vygotsky. In H. Daniels, M. Cole, \& J.V. Werstch (Eds.), The Cambridge Companion to Vygotsky (pp. 276-303). New York: NY: Cambridge University Press.

Donald, M. (2001). A mind so rare. New York, NY: W.W. Norton \& Company. 
Echevarria, J., \& Graves, A. (2011). Sheltered content instruction: Teaching english learners with divers abilities. Boston, MA: Allyn \& Bacon.

Echevarria, J., Short, D., \& Vogt, M. (2000). Making content comprehensible for english language learners:The SIOP model. Boston, MA: Allyn \& Bacon.

Faraco, M., \& Kida, T. (2008). Gesture and the negotiation of meaning in a second language classroom. In S. McCafferty \& G. Stam (Eds.), Gesture: Second language acquisition and classroom research (pp. 280-297). New York, NY: Routledge.

Geertz, C. (1973). The interpretation of cultures. New York, NY: Basic Books

Gibson, J.J. (1979). The ecological approach to visual perception. Boston, MA: Cornell University.

Goodman, N. (1968). The languages of art: An approach to a theory of symbols. Indianapolis, IN: Hackett.

Gullberg, M. (1998). Gesture as a communication strategy in second language discourse: A study of learners of French and Swedish. Lund, Sweden: Lund University Press.

Gullberg, M., \& McCafferty, S. (2008). Introduction to gesture and SLA: Toward an integrated approach. Studies in Second Language Acquisition, 3033. 146.

Haught, J., \& McCafferty, S. (2008). Embodied language performance: Drama and the ZPD in the second language classroom. In M. Poehner \& J. Lantolf (Eds.), Sociocultural theory and the teaching of second languages. London, UK: Equinox.

James, W. (1890). Principles of psychology. New York, NY: Dover Publications.

Kendon, A. (1994). Do gestures communicate? A review. Research on language and social interaction, 27(3), 175-200.

Kida, T. (2008). Does gesture aid discourse comprehension in the L2? In S. McCafferty \& G. Stam (Eds.), Gesture: Second language acquisition and classroom research (pp. 131-156). London, UK: Routledge.

Kramsch, C. (2008). Ecological perspectives on foreign language education. Language Teaching, 41(3), 389-408.

Lantolf, J. (2000). Introducing sociocultural theory. In J. Lantolf (Ed.), Sociocultural theory and second language learning (pp. 1-26). Oxford, UK: Oxford University Press.

Lantolf, J., \& Thorne, S. (2006). Sociocultural theory and the genesis of second language development. Oxford, UK: Oxford University Press.

Lazaraton, A. (2004). Gesture and speech in the vocabulary explanations of one ESL teacher: A microanalytic inquiry. Language Learning, 54 (1), 79-117.

Lindblom, J., \& Ziemke, T. (2007). Embodiment and social interaction: A cognitive science perspective. In T. Ziemke, J. Zlatev, \& R. Frank (Eds.), Body, language and mind: Vol 1. Embodiment (pp. 129-163). Berlin, Germany: Mouton de Gruyter.

McCafferty, S. (1998). Nonverbal expression and L2 private speech. Applied Linguistics, 19, 73-96.

McCafferty, S. (2008a). Mimesis and second language acquisition. Studies in Second Language Acquisition, 30, 147-167.

McCafferty, S. (2008b). Material foundations for second language acquisition: Gesture, metaphor, and internalization. In S. McCafferty \& G. Stam, (Eds.), Gesture: Second language acquisition and classroom research (pp. 47-65). London, UK: Routledge. 
McCafferty, S., \& Stam, G. (2008). Gesture: Second Language Acquisition and Classroom Research. New York, NY: Routledge.

McNeill, D. (1992). Hand and mind. Chicago, IL: Chicago University Press.

McNeill, D. (2005). Gesture and thought. Chicago, IL: University of Chicago Press.

McNeill, D. (2012). How language began: Gesture and speech in human evolution. Cambridge, UK: Cambridge University Press.

Merleau-Ponty, M. (1962). Phenomenology of perception (C. Smith, trans.). London, UK: Routledge.

Rosborough, A. (2012). Teacher and students' use of gesture as a meaning-making affordance for second language learning. In B. Yoon \& H.K. Kim (Eds.), Teachers' roles in second language learning: Classroom applications of sociocultural theory (pp. 63-80). Charlotte, N.C.: Information Age Publishing.

Sime, D. (2006). What do learners make of teachers' gestures in the language classroom? International Review of Applied Linguistics in Language Teaching, 44 (2), 211-230.

Stam, G. (2008). What gestures reveal about second language acquisition. In S. McCafferty \& G. Stam (Eds.), Gesture: Second language acquisition and classroom research (pp. 231-255). London, UK: Routledge.

Thibault, P. (2004). Brain, mind, and the signifying body. London, UK: Continuum. Thibault, P. (2011). First-order languaging dynamics and second-order language: The distributed language view. Ecological Psychology, 23 (3), 210-245.

Tomasello, M. (2008). Origins of human communication. Cambridge, MA: MIT Press. Valsiner, J. (1997). Culture and the development of children's action. New York, NY:Wiley

van Lier, L. (1996). Interaction in the language curriculum: Awareness, autonomy, \& authenticity. New York, NY: Longman Publishing.

van Lier, L. (2004). The ecology and semiotics of language learning: A sociocultural perspective. Boston, MA: Kluwer Academic Publishers.

van Lier, L. (2008). The ecology of language learning and sociocultural theory. In A. Creese, P. Martin, P., \& N. H. Hornberger (Eds.), Encyclopedia of language and education: Vol. 9. Ecology of language (2nd ed., pp. 53-65). Boston, MA: Springer.

Vygotsky, L. (1978). Mind in society: The development of higher psychological processes. Cambridge, MA: Harvard University Press.

Vygotsky, L. (1986). Thought and language. Cambridge, MA: MIT press.

Vygotsky, L. (1924/1997a). The collected works of L.S. Vygotsky: Vol. 3. Problems of the theory and history of psychology (R.W. Reiber \& A.S. Carton, Eds.). New York NY: Plenum Press.

Vygotsky, L. (1997b). The collected works of L. S. Vygotsky: Vol. 4. History of the development of higher mental functions (R.W. Reiber \& A. S. Carton, Eds.). New York, NY: Plenum Press.

Wells, G. (1999). Dialogic inquiry: Towards a sociocultural practice and theory of education. Cambridge, UK: Cambridge University Press.

Wells, G. (1986/2009). The meaning makers: Children learning language and using language to learn. Portsmouth, NH: Heinemann.

Wertsch, J.V. (1985). Vygotsky and the social formation of mind. Cambridge, MA: Harvard University Press. 
Wertsch, J.V. (1991). Voices of the mind: A sociocultural approach to mediated action. Cambridge, MA: Harvard University Press.

Wertsch, J.V. (1998). Mind as action. New York, NY: Oxford University Press.

Zlatev, J. (2007). Embodiment, language, and mimesis. In T. Ziemke, J. Zlatev, \& R. Frank (Eds.), Body, language and mind: Vol 1. Embodiment (pp. 297-337). Berlin, Germany: Mouton de Gruyter.

Zazzo, R. (1968). Conduites et conscience: Vol. 1. Psychologie de l'enfant et method genetique. SUI, Neuchatel: Delachaux et Niestle.

\section{Authors:}

Alessandro (Alex) Rosborough, Ph.D.

Teacher Education Department

206-S McKay School of Education

Brigham Young University

Provo

UT 84602

USA

email: alex_rosborough@byu.edu

\section{APPENDIX A TRANSCRIPTION DATA DESCRIPTION}

A transcription code modified and based on McNeill (1992).

1. Speech is transcribed fully from the videotape in ordinary orthography

2. Gesture is typed in italics below the speech. Gesture codes include the following:

[ ] gesture phrase (stroke in boldface)

/ silent pause (multiple slashes for longer pauses)

(///) Stroke not associated with speech (sometimes ellipsis)

$\wedge \quad$ Rise in voice intonation

$\backslash \quad$ Fall in voice intonation

( ) Additional information to provide context to the situation by the researcher

Numbers were assigned to every speech turn performed by the participant. Although transcriptions were made for entire scenes, some examples only demonstrate partial dialogues. "Skips" are noted in the transcriptions. It should also be noted that extended speech is segmented by conventional commas and periods to best match the pauses and meaning of the participant's speech. However, that periods and commas are not a part of speech and they are placed according to this researcher's understanding of the dialogue. 
3. Gestures were analyzed according to the following:

1. Identification of the movements that are gestures (particularly the hands but also the arm, head, and body movements)

2. Identification of the stroke phase, and in some cases the preparation or retraction phases (concentration on the trajectory, shape, and posture)

3. Location of the boundaries of the gesture phases in the relevant part of the phonological transcription

4. Codings for gesture types include the following:

5. Representational (represents attributes, actions, or relationships of objects or characters); two kinds-(1) Iconic (2) Metaphoric

6. Deictic (finger points or other indications of either concrete or imaginary objects or people)

7. Beats (formless hands that convey no information but move in rhythmic relationship to speech - category confirmed by beat filter below)

8. Emblems/Italianate (deliberate and standardized movements that have a direct verbal equivalent known to others in the same speech community, typically demonstrating continually the same meaning when performed) 